\title{
Assessing phenotypic and polygenic models of ADHD to identify mechanisms of risk for longitudinal trajectories of externalizing behaviors
}

\author{
James J. Li \\ Department of Psychology and Waisman Center, University of Wisconsin-Madison, Madison, WI, USA
}

\begin{abstract}
Background: Children with ADHD frequently engage in higher rates of externalizing behaviors in adulthood relative to children without. However, externalizing behaviors vary across development. Little is known about how this risk unfolds across development. Phenotypic and polygenic models of childhood ADHD were used to predict individual differences in adult externalizing trajectories. Supportive parenting, school connectedness, and peer closeness were then examined as causal mechanisms. Methods: Data were from the National Longitudinal Study of Adolescent to Adult Health $(N=7,674)$. Externalizing behavior was measured using data from age 18 to 32 and modeled using latent class growth analysis. Child ADHD was measured using retrospective self-report (phenotypic model) and genome-wide polygenic risk scores (polygenic model). Multiple mediation models examined the direct and indirect effects of the phenotypic and polygenic models (separately) on externalizing trajectories through the effects of adolescent supportive parenting, school connectedness, and peer closeness. Results: Phenotypic and polygenic models of ADHD were associated with being in the High Decreasing (3.2\% of sample) and Moderate (16.1\%) adult externalizing trajectories, but not the severe Low Increasing trajectory $(2.6 \%)$, relative to the Normal trajectory (78.2\%). Associations between both models of ADHD on the High Decreasing and Moderate trajectories were partially mediated through the effects of school connectedness, but not supportive parenting or peer closeness. Conclusions: Findings shed light on how childhood ADHD affects downstream psychosocial processes that then predict specific externalizing outcomes in adulthood. They also reinforce the importance of fostering a strong school environment for adolescents with (and without) ADHD, as this context plays a critical role in shaping the development of externalizing behaviors in adulthood. Keywords: ADHD; molecular genetics; antisocial behavior; longitudinal studies; mediation.
\end{abstract}

\section{Introduction}

Children with attention-deficit/hyperactivity disorder (ADHD) are more likely to be arrested, convicted or incarcerated as adults, even in the absence of childhood conduct disorder (Storebø \& Simonsen, 2016). Yet, not all children with ADHD develop adult externalizing behaviors (Mordre, Groholt, Kjelsberg, Sandstad, \& Myhre, 2011). Relatively little is known about how psychosocial processes also contribute to its development over time. A better understanding about how these processes unfold may lead to more precise or novel interventions for this vulnerable population.

The progression from childhood ADHD to adult externalizing behaviors is believed to result from common heritable mechanisms (Beauchaine, Hinshaw, \& Pang, 2010; Lahey, Zald, Hakes, Krueger, \& Rathouz, 2014). Twin studies suggest that a common latent factor accounts for a portion of the covariation among ADHD and other externalizing disorders (Rhee, Lahey, \& Waldman, 2015; Tuvblad, Zheng, Raine, \& Baker, 2009). Genome-wide association studies (GWAS) have also shown that common single nucleotide polymorphisms (SNPs) account for $38 \%-44 \%$ of the heritability of a common latent factor (Neumann et al., 2016). In fact, an aggregate of the common genetic variants for ADHD (i.e.,

Conflict of interest statement: No conflicts declared. polygenic risk scores; PRS) explained more variance in the externalizing dimension relative to the internalizing dimension (Brikell et al., 2018). Riglin et al. (2016) also found that PRS for ADHD was associated with 'multimorbidity,' including for conduct problems and neurodevelopmental traits (Riglin et al., 2016). Collectively, results suggest that a multitude of risk variants for ADHD may increase liability to the development of externalizing behaviors in later life.

Notably, there are individual differences in how externalizing behaviors develop during adulthood (Li, 2017; Moffitt, Caspi, Harrington, \& Milne, 2002). Studies typically converge on at least two externalizing trajectories: life-course persistent (i.e., physical aggression and rule-breaking behaviors, beginning at an early age and persisting into early adulthood) and adolescence-limited (i.e., less severe antisocial behaviors that peak during adolescence and desist by early adulthood) (Moffitt et al., 2002). However, other studies identified several other externalizing trajectories that are unique to adulthood (Li, 2017; Sampson \& Laub, 2003). Given that not all individuals with ADHD develop adult externalizing behaviors (Storebø \& Simonsen, 2016), it is surprising that no studies have examined whether this association might vary based on individual differences in the adult externalizing trajectories.

Psychosocial factors may help to explain the association between childhood ADHD and 
prospective externalizing behaviors. For instance, negative parenting behaviors such as poor supervision and communication, lack of involvement, and timid discipline predict more childhood externalizing problems, and childhood externalizing problems also prospectively predicts more negative parenting by adolescence (Burke, Pardini, \& Loeber, 2008). Subsequently, adolescents who feel unsupported and distant from their parents have a higher risk of engaging in adult externalizing behaviors (Bosco, Renk, Dinger, Epstein, \& Phares, 2003; Li, 2017). Evidence suggests the reciprocity of child ADHD and subsequent parental behavior may be genetically mediated, as genetic influences on child ADHD (measured by the biological mother's ADHD) evoked more hostility from the adoptive mother toward her child at a later time (Harold et al., 2013). Thus, children with ADHD may evoke less supportive parenting by the time they reach adolescence, which in turn may increase their risk of engaging in adult externalizing behaviors.

Another psychosocial mechanism that may explain the association between childhood ADHD and adult externalizing behaviors is the school environment (McNeely, Nonnemaker, \& Blum, 2002). For instance, school connectedness reflects the extent to which adolescents feel accepted and supported by others in their school environment, and predicts lower levels of delinquency and alcohol use (Crosnoe, Erickson, \& Dornbusch, 2002; Li, 2017). Adolescents who felt less connected to their schools or had poor relationships with their teachers were more likely to engage in delinquency during adolescence and have more substance use and mental health problems in adulthood (Jacobson \& Rowe, 1999; Shochet, Dadds, Ham, \& Montague, 2006). Peers may also play a central role in shaping risky behaviors during adolescence (Albert, Chein, \& Steinberg, 2013; Mrug \& Windle, 2009). Strong peer relationships during adolescence may protect against the development of later internalizing and externalizing outcomes (Jager, Yuen, Putnick, Hendricks, \& Bornstein, 2015), but two studies found either no association (Heiman, 2005) or an opposite association (Glass, Flory, \& Hankin, 2012). More studies are needed to determine how childhood ADHD affects adolescent peer relationships and subsequent externalizing outcomes, while also accounting for the broader school context. It is plausible that children with ADHD report fewer close friends by adolescence, which in turn may increase their risk of engaging in adult externalizing behaviors.

This study examines whether associations between childhood ADHD and trajectories of adult externalizing behaviors from age 18 to 32 could be explained by environmental experiences during adolescence (i.e., supportive parenting, school connectedness, and peer closeness) via mediation using prospective longitudinal data from the National Longitudinal Study of Adolescent to Adult Health (Add Health). This study tested two distinct models of childhood ADHD to establish mediation. The first was a phenotypic model in which childhood symptom counts of ADHD were assessed retrospectively. The second was a polygenic model (as a PRS), which accounts for the possibility of common method variance (Lindell \& Whitney, 2001).

\section{Methods \\ Participants}

Add Health began in 1994 and ascertained a stratified random sample of adolescents in grades 7-12 from high schools across the U.S. Data were collected from adolescents, parents, fellow students, school administrators, siblings, friends and romantic partners across four waves of data collection: wave I (19941995 , grades $7-12, N=20,745)$, wave II (1995-1996, grades 8$12, N=14,738)$, wave III (2001-2002, ages $18-26, N=15,197)$, and wave IV (2007-2008, ages 24-32, $N=15,701)$. Genotypes were available for 9,128 individuals, but the current analyses were performed for individuals in genotypic and phenotypic information germane to the current investigation were available, resulting in an analytic sample size of 7,674 . Overall, $46 \%$ of this sample was male, and the racial-ethnic composition of the genotypic sample was $63.2 \%$ Caucasian, $21.2 \%$ African-American, 5.1\% Asian, and 10.6\% Hispanic. Other demographic information (e.g., highest parental education and household income at wave I) is available in the Supporting Information online (Table S1). The University's Institutional Review Board approved this study.

\section{Measures}

Externalizing behavior. Adult externalizing behaviors were assessed during in-home interviews conducted at waves III and IV (spanning ages 18-32). Thirteen items were assessed at each wave, reflecting the presence of non-aggressive rulebreaking behaviors (e.g., property damage, stealing, selling drugs), aggressive behaviors (e.g., pulling a knife or gun on someone, engaging in a fight), and substance use behaviors (e.g., frequency of cigarette smoking, binge drinking, and marijuana use, in the past 30 days). All items were dichotomized to reflect the presence or absence of each behavior. Items were then summed to create a composite score at each wave (Cronbach's $\alpha=.66$ and .56 for waves III and IV, respectively). ${ }^{1}$

$A D H D$. Childhood ADHD symptoms were assessed retrospectively during the in-home interview at wave III using 17 of the 18 items for ADHD according to the Diagnostic and Statistical Manual of Mental Disorders (American Psychiatric Association, 2013). Items were rated on a 4-point Likert scale regarding how often the symptom 'best describes your behavior when you were [between 5 and 12].' The item was then dichotomized to indicate the presence (i.e., often or very often response) or absence (i.e., never or sometimes response) of the symptom, and summed to create a composite score for ADHD symptoms (Cronbach's $\alpha=.86$ ).

Supportive parenting. Supportive parenting was assessed during wave I using 12 items related to maternal warmth (e.g., 'your mother is warm and loving toward you'), care (e.g., 'how much do you feel that your parents care about you?'), closeness (e.g., 'how close do you feel to your mother?'), communication quality (e.g., you are satisfied with the way your mother you and you communicate with each other'), understanding (e.g., "how much do you feel that people in your family understand you?') and the overall quality of the parental relationship (e.g., 'overall, you are satisfied with your relationship with you mother'). This construct has been used by 
several previous Add Health studies, demonstrating good internal consistency (Cronbach $\alpha=.83$ ) and predictive validity (Li, Berk \& Lee, 2013). All 12 items were rated on a five-point scale, where $0=$ not at all and $4=$ very much. Scores for each item were summed to create a composite score.

School connectedness. Six items from wave I assessed feelings of belongingness (e.g., 'you feel close to people at your school,' 'you feel like you are part of your school,' 'you feel close to people at school'), teacher support (e.g., 'your teachers care a lot about you,' 'teachers treat students fairly'), and safety (e.g., 'you feel safe in your school'). Items pertaining to peer closeness were not included. All items were rated on a five-point scale, where $0=$ strongly disagree and $4=$ strongly agree. The school connectedness scale demonstrates good predictive validity in previous Add Health studies (Batanova \& Loukas, 2014; McNeely et al., 2002). The scale also has adequate internal consistency in the sample (Cronbach $\alpha=.73$ ). Scores for each item were summed and the resultant score was then standardized.

Peer closeness. At wave I, participants were asked to nominate up to five male and five female friends. For each nominated friend, participants were asked five yes or no followup questions regarding the closeness of that relationship in the past 7 days, such as whether the participant visited the friend's home, spent time or went somewhere with them, or felt comfortable in talking to them about a problem. A total score was calculated based on the sum of each positive endorsement for each friend, male or female, that was nominated. This score was then standardized. A previous Add Health study showed good predictive validity for this variable with respect to negative adult outcomes (Carbonaro \& Workman, 2013).

Genotyping and quality control. Saliva were obtained from participants at wave IV. Genotyping was done on the Omni1-Quad BeadChip and the Omni2.5-Quad BeadChip. Add Health European genetic samples were imputed on Release 1 of the Human Reference Consortium (HRS r1.1). Non-European samples were imputed using the 1000 Genomes Phase 3 reference panel. Of 606,673 variants, 13,721 were removed with a per-variant missing call rate filter of 0.02 ; 245,589 were removed with a Hardy-Weinberg Equilibrium filter of 0.0001 , and 609 were removed with a minor allele frequency filter of 0.01 , leaving 346,754 SNPs carried through to imputation. Additional details of the quality control are available online (https://www.cpc.unc.edu/projects/addhea lth/documentation/guides).

Polygenic Risk Scores (PRS). Polygenic Risk Scores are the weighted sum of the regression coefficient for each SNP from an independent GWAS of ADHD and allele frequencies for the same SNPs in the Add Health genome-wide data. This study used the most recent GWAS for ADHD (Demontis et al., 2019), which included 55,374 individuals $(20,183$ cases and 35,191 controls) from 12 studies of mixed ancestries and a replication sample of 93,916 individuals from two mixed ancestry cohorts. PRS were computed using all the available SNP information and standardized within ancestry groups in Add Health (Braudt \& Harris, 2018). Additional controls for population stratification were done by incorporating the first 10 ancestry-specific principal components (PC) of the genetic data per expert recommendations (Price et al., 2006).

\section{Data analysis}

Step 1. Latent class growth (LCG) models were fit to characterize externalizing behavior trajectories from wave III to IV. Data were restructured such that time was represented by age (18-32) rather than by wave. Missingness was accounted for by full information maximum likelihood estimation in Mplus
7.4 (Muthén \& Muthén, 2015). A zero-inflated model was specified given the positive skew of the externalizing outcomes at each age. The form factor of the LCG model was identified by comparing three models: (a) intercept, (b) intercept and slope, and (c) intercept, slope and quadratic. Models that had lower Bayesian Information Criterion (BIC) values indicate better fit to the data, although models were also evaluated based on their interpretability. Following the selection of the best fitting form factor, similar model fitting procedures were used to identify the optimal number of latent classes representing externalizing trajectories of that form factor.

Step 2. After determining the best fitting model from Step 1, multinomial logistic regressions were conducted to examine associations of the phenotypic and polygenic models of ADHD on latent class membership. The reference class was most prevalent class (i.e., a 'normal,' or a low externalizing class).

Step 3. Posthoc multiple mediator models (Hayes, 2013) were fit to examine the simultaneous effects of supportive parenting, school connectedness, and peer closeness (measured during adolescence at wave I) as mediators in the association between the phenotypic and polygenic models of ADHD and the externalizing class trajectories (measured at waves III and IV). In multiple mediation, the direct effect, $c$ ', reflects the pathway of both models of ADHD to the odds of being in the externalizing trajectory class (relative to a normal class) independent of the mediational effects of the supportive parenting, school connectedness, and peer closeness, $a_{i}$ estimates the effect of both models of ADHD on each mediator, and $b_{i}$ estimates the effect of each mediator on the odds of the externalizing class trajectory. As there are three mediators in each model, specific indirect effects of each mediator on externalizing classes reflect each of these individual pathways (i.e., childhood ADHD $\rightarrow$ adolescent psychosocial mediator $\rightarrow$ odds of being in an adult externalizing class relative to normal class) while accounting for the shared associations between them (Hayes, 2013). The total effect, $c$, reflects the association of both models of ADHD on the odds of being in an externalizing class, not accounting for the effects of any mediators. All models controlled for demographic variables (i.e., sex, age, highest level of education, income) and ancestry based on PCs of the genetic data.

\section{Results Preliminary analyses}

Descriptive statistics and bivariate correlations are presented in Tables $\mathrm{S} 1$ and $\mathrm{S} 2$.

\section{Externalizing trajectories}

Class comparisons in the LCG analysis indicated that a four-class linear solution was an optimal fit to the data, in part due to its superior fit statistics $(\mathrm{BIC}=88859.70 ;$ entropy $=.83 ;$ LRT $p$-value comparing five to four class $=<.001$ ) (Table S3), but also due to the clarity of interpretation of the classes relative to other class models (see Figure 1). Results for the model fit statistics are in the Tables S3 and S4. In the four-class model, 'Normal' (78\% of sample) consisted of individuals who showed consistently low levels of externalizing behavior from age 18 to 32 . 'High Decreasing' (3.2\%) characterized individuals who had relatively high levels of externalizing behavior at age 18 but desisted in these behaviors such that by age 32 , they were virtually indistinguishable 


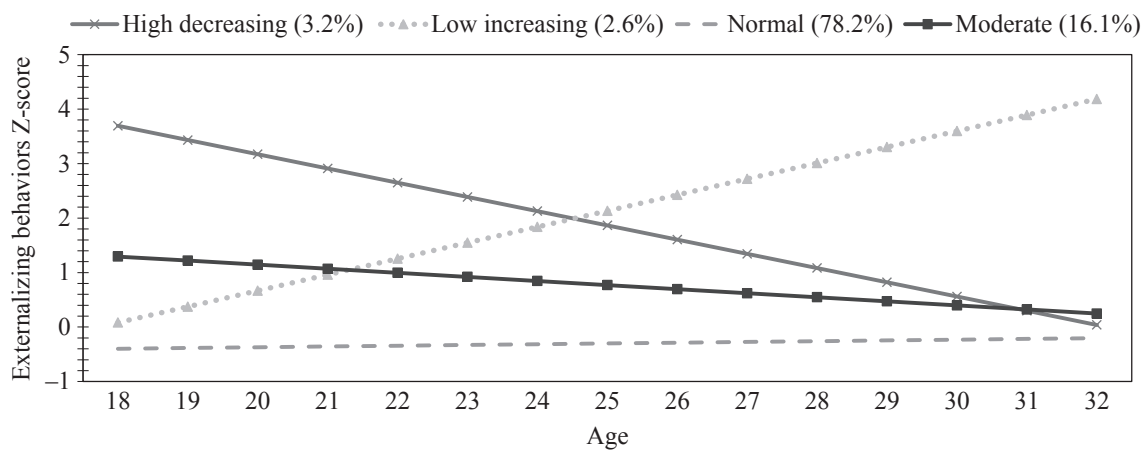

Figure 1 Trajectories and prevalence rates of adult externalizing behaviors in Add Health

from 'Normal' individuals. Relative to Normal individuals, 'Moderate' (16.1\%) individuals showed slightly higher rates of externalizing behavior age at 18 , and maintained moderate levels of externalizing behaviors throughout adulthood, although these individuals were also virtually indistinguishable from Normal individuals by age 32. Finally, a 'Low Increasing' class $(2.6 \%)$ emerged, representing a subset of individuals who showed relatively low levels of externalizing behavior at age 18 but significantly increased in their externalizing behavior through their $20^{\prime} \mathrm{s}$ and into their early $30^{\prime} \mathrm{s}$.

\section{Phenotypic and polygenic ADHD $\rightarrow$ Adult externalizing trajectories}

Associations between childhood ADHD symptoms (i.e., phenotypic model) and externalizing trajectories were examined in a multinomial logistic regression, with the Normal class serving as the reference category (Table S5). Childhood ADHD symptoms predicted $12.1 \%$ increased odds in the High Decreasing $(95 \% \mathrm{CI}=1.081,1.161)$ and $4.3 \%$ increased odds in the Moderate $(95 \% \mathrm{CI}=1.024,1.064)$ externalizing trajectories, but was not associated with the Low Increasing $(95 \% \mathrm{CI}=.962,1.070)$ trajectory, relative to the Normal trajectory.

This pattern of association was entirely consistent in the polygenic model (Table S5). PRS for ADHD predicted $17.0 \%$ increased odds in the High Decreasing $(95 \% \mathrm{CI}=1.002,1.366)$ and $8.0 \%$ increased odds in the Moderate $(95 \% \mathrm{CI}=1.004,1.163)$ externalizing trajectories, but was not associated with the Low Increasing $(95 \% \mathrm{CI}=0.868,1.265)$ trajectory, relative to the Normal trajectory.

\section{Phenotypic and polygenic ADHD $\rightarrow$ Adolescent mediators $\rightarrow$ High Decreasing trajectory}

Multiple mediation models were fit to examine the indirect effects of the phenotypic and polygenic models of ADHD on each externalizing trajectory for which associations were significant in the previous models (i.e., High Decreasing vs. Normal, Moderate vs. Normal), through the simultaneous effects of supportive parenting, school connectedness, and peer closeness.
Figure 2A shows the regression paths of the phenotypic model examining ADHD symptoms on the odds of being in the High Decreasing trajectory (relative to Normal) through the mediators. After accounting for the mediators, ADHD symptoms predicted 9.7\% increased odds $(95 \% \mathrm{CI}=1.068,1.161)$ of being in the High Decreasing trajectory relative to Normal. However, the indirect effect of ADHD symptoms on this trajectory was only significant through the effects of school connectedness, but not supportive parenting or peer closeness (Table 1).

This result was consistent in the polygenic model (Figure 2B). In fact, there was no longer evidence of association between PRS on High Decreasing trajectory membership after accounting for the mediators (95\% CI $=0.914,1.318)$. The indirect effect of PRS on this trajectory was only significant through the effects of school connectedness, but not supportive parenting or peer closeness.

\section{Phenotypic and polygenic ADHD $\rightarrow$ Adolescent mediators $\rightarrow$ Moderate trajectory}

Next, Figure 3A shows the regression paths of the phenotypic model examining ADHD symptoms on the odds of being in the Moderate trajectory (relative to Normal) through the mediators. After accounting for the mediators, ADHD symptoms predicted only $3.3 \%$ increased odds $(95 \% \mathrm{CI}=1.010,1.057)$ of being in the Moderate trajectory relative to Normal. However, the indirect effect of ADHD symptoms on this trajectory was significant through the effects of school connectedness and supportive parenting, but not peer closeness (see Table 1).

In the polygenic model (Figure 3B), there was no longer evidence of association between PRS and Moderate trajectory membership after accounting for the mediators $(95 \% \mathrm{CI}=0.973,1.145)$. However, the indirect effect of PRS on this trajectory was only significant through the effects of school connectedness, but not supportive parenting or peer closeness.

\section{Sensitivity analysis}

To determine whether the mediation models could be explained by other factors, sensitivity analyses were 


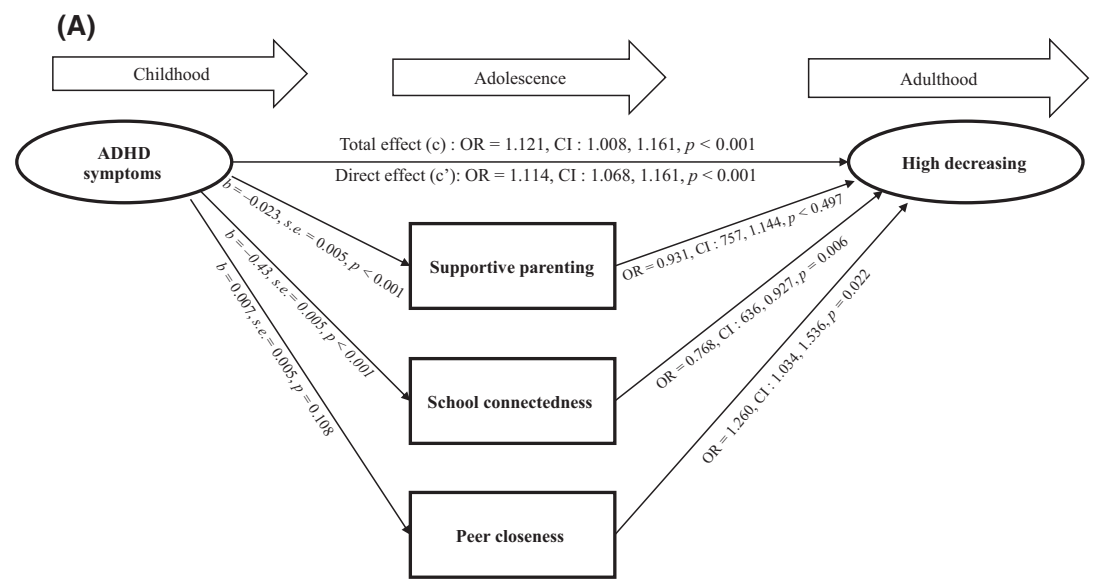

(B)

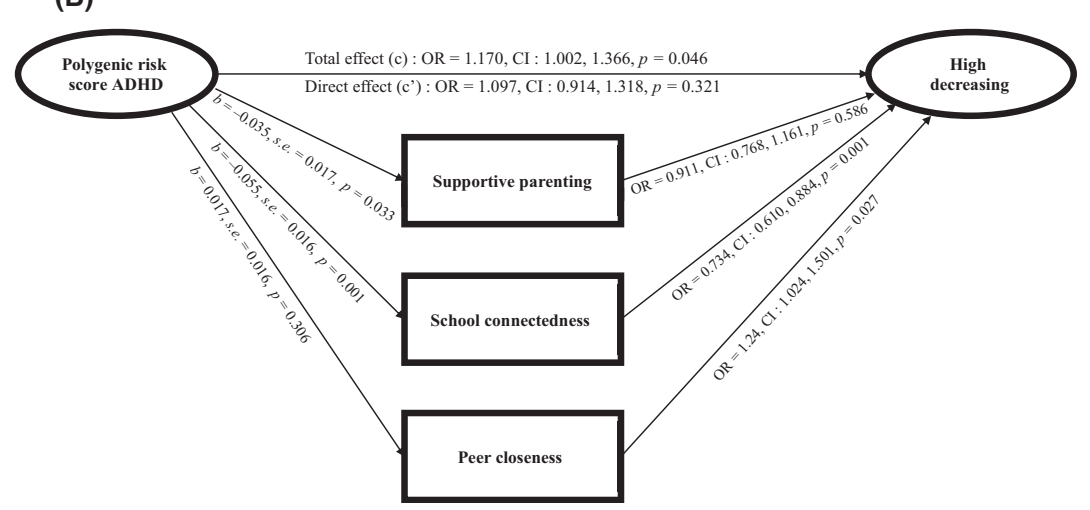

Figure 2 Direct and indirect effects of ADHD on odds of being in the High Decreasing externalizing trajectory relative to the Normal trajectory. (A) Phenotypic model; (B) Polygenic model

Table 1 Total and specific indirect effects of the phenotypic and polygenic models of ADHD

\begin{tabular}{|c|c|c|c|c|}
\hline & \multicolumn{2}{|c|}{ Phenotypic model } & \multicolumn{2}{|c|}{ Polygenic model } \\
\hline & \multicolumn{2}{|c|}{$95 \% \mathrm{CI}$} & \multicolumn{2}{|c|}{$95 \% \mathrm{CI}$} \\
\hline \multicolumn{5}{|l|}{ High decreasing trajectory vs. normal trajectory } \\
\hline Total indirect effects & .008 & .024 & .008 & .043 \\
\hline ADHD $\rightarrow$ Supportive parenting $\rightarrow$ High decreasing & -.003 & .008 & -.005 & .015 \\
\hline ADHD $\rightarrow$ School connectedness $\rightarrow$ High decreasing & .003 & .020 & .006 & .036 \\
\hline ADHD $\rightarrow$ Peer closeness $\rightarrow$ High decreasing & 0 & .006 & -.002 & .017 \\
\hline \multicolumn{5}{|l|}{ Moderate trajectory vs. normal trajectory } \\
\hline Total indirect effects & .009 & .019 & .005 & .030 \\
\hline ADHD $\rightarrow$ Supportive parenting $\rightarrow$ Moderate & .003 & .009 & 0 & .012 \\
\hline ADHD $\rightarrow$ School connectedness $\rightarrow$ Moderate & .003 & .011 & .004 & .019 \\
\hline ADHD $\rightarrow$ Peer closeness $\rightarrow$ Moderate & 0 & .004 & -.004 & .010 \\
\hline
\end{tabular}

Bold pathways represent statistically significant indirect effects that were replicated by both the phenotypic and polygenic models of ADHD.

conducted by examining the indirect effects of the phenotypic and polygenic models of ADHD on externalizing trajectories via the effects of adolescent sensation seeking, a key marker of risk for adult substance use and externalizing outcomes and a putative intermediate phenotype (Li et al., 2017). Controlling for the same covariates, the models were completely consistent with the main findings. That is, the effects of ADHD symptoms and PRS on externalizing trajectories were either fully or partial mediated via the effects of school connectedness, but not sensation seeking, supportive parenting, or peer closeness (see Table S6).

\section{Discussion}

Phenotypic and polygenic models of ADHD were used to examine associations between ADHD and adult externalizing behavior trajectories measured from age 18 to 32 in Add Health. In the phenotypic model, childhood ADHD symptoms prospectively predicted increased odds of being in either the High 


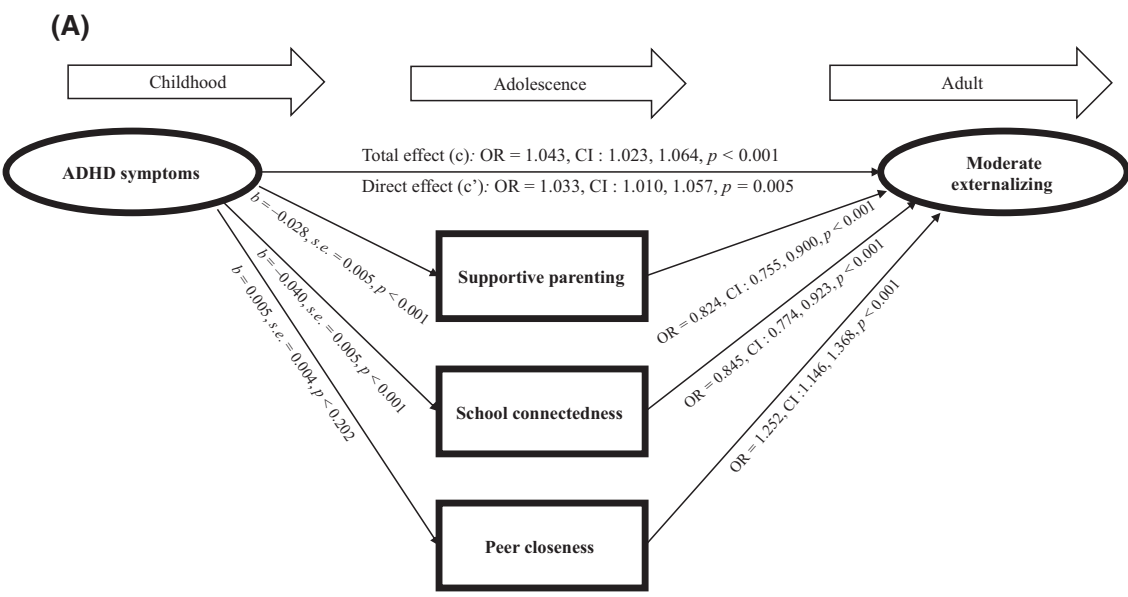

(B)

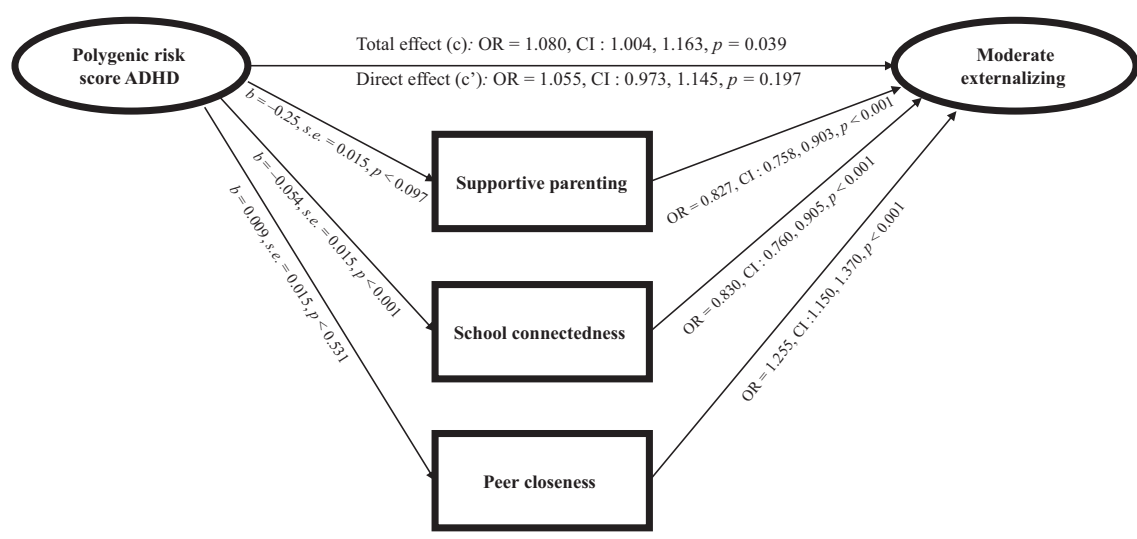

Figure 3 Direct and indirect effects of ADHD on odds of being in the Moderate externalizing trajectory relative to the Normal trajectory. (A) Phenotypic model; (B) Polygenic model

Decreasing or Moderate externalizing trajectories (compared to Normal), but not Low Increasing. Furthermore, both of these associations were partially mediated via the effects of school connectedness during adolescence, but not supportive parenting or peer closeness. These patterns of associations were replicated in the polygenic model, suggesting that the current results are robust and unlikely confounded by common method variance.

The four-classes of externalizing trajectories were fairly consistent with other studies that focused on adults (Li, 2017; Nagin \& Tremblay, 2005), particularly in showing the continuity and heterogeneity of externalizing behaviors when data extend to later adulthood. Crucially, phenotypic and polygenic models of ADHD were only associated with the High Decreasing and Moderate externalizing trajectories, and not with the more severe, Low Increasing trajectory. This is notable because both High Decreasing and Moderate trajectories were indistinguishable from the Normal trajectory by age 32 , suggesting that the heterotypic continuity observed among the subset of children with ADHD who persist in externalizing behaviors in adulthood may desist in these behaviors by their mid-30's. This finding helps to explain the inconsistent associations reported in the literature (Mordre et al., 2011; Storebø \&
Simonsen, 2016), which had not modelled externalizing behavior over time but focused on diagnostic status or criminal offenses at a particular point in adulthood. Furthermore, the lack of association between phenotypic and polygenic models of ADHD with the more severe Low Increasing trajectory suggests unique etiological mechanisms underlying both phenotypes. It is also possible that the link between childhood ADHD and more severe externalizing behavior may depend on the co-occurrence of a childhood conduct disorder (CD), which some have suggested should constitute a unique subtype given its neurobiological features differ from other subtypes (Banaschewski et al., 2003).

Another key finding was the robust effects of school connectedness as a potential psychosocial mechanism underlying the association of childhood ADHD and High Decreasing and Moderate externalizing trajectories, even after accounting for the simultaneous effects of supportive parenting and peer closeness. This provides preliminary support that children with ADHD may have a harder time feeling accepted or connecting with others at their schools, resulting in a higher likelihood of engaging in externalizing behaviors during part of their adult years. Other studies have similarly shown that even when comorbid psychopathology (Shochet et al., 2006) and 
peer and familial factors ( $\mathrm{Li}, 2017)$ were accounted for, school connectedness continues to account for a significant portion of the variance in later mental health problems. Notably, results suggest a geneenvironment correlation with respect to PRS and school connectedness. Correlations between genotype and environment confound interpretations of geneenvironment interaction (Knafo \& Jaffee, 2013), which are far more studied in the context of geneticallyinformed investigations that integrate psychosocial measures of the environment. Given the complexity of the genetic architecture for ADHD, genetically-informed studies should focus on elucidating other pathways from genes to behavior, as these studies may help to uncover new targets for interventions.

The results should be interpreted in light of some limitations. Childhood ADHD symptoms were assessed retrospectively, which may be confounded by recall bias. However, a key strength of the study is that a polygenic model of ADHD was also tested, providing an unbiased approach to characterizing the underlying genetic liability to ADHD using only participantgeneticinformation. Convergence between the results of the phenotypic and polygenic models provided support that pathways from childhood ADHD tolater externalizing behaviors were not entirely driven by methodological confounds, although they could not be ruled out. Another limitation is that psychosocial influences were examined at wave 1 , when participants were adolescents in high school (which provided complete temporal separation between the mediators and the outcomes). These measures were not assessed in childhood, which would have provided the opportunity to measure the change in environment over time as well. Furthermore, some of the indirect effects were small, highlighting the multifactorial nature of mental health outcomes. Although both models of ADHD and adult antisocial trajectories were still significantly attenuated when accounting for the effects of school connectedness (and controlling for parenting, peer effects, and trait sensation seeking), there are a multitude of other risk (and protective) pathways stemming from ADHD to adult trajectories of externalizing behaviors that were not examined in the current study. Finally, given the high degree of age heterogeneity at each wave, there may have been potential 'cohort effects' that could not have been directly measured.

This study rigorously and innovatively combines both phenotypic and polygenic approaches to shed light on how childhood ADHD affects downstream psychosocial processes that then predict specific externalizing outcomes in adulthood. Future studies of psychiatric phenotypes should continue to exploit increasingly accessible genome-wide datasets to validate other (e.g., biological) mechanisms of risk, especially given that the use of PRS models have ascended rapidly in recent years but little is known about how these signals eventuate into behavioral outcomes. These findings also have clinical implications. Childhood ADHD symptoms may lead to increased feelings of disconnect once the child enters or progresses through high school, a key risk factor in predicting subsequent adult externalizing behavior. There have been calls by experts for a greater focus on enhancing school connectedness as a potential target for preventing negative mental health outcomes in later life (e.g., Li, 2017; Shochet et al., 2006). For instance, McNeely et al. (2002) suggested that improving classroom climates, offering more extracurricular activities, introducing more tolerant disciplinary policies, and reducing classroom sizes may potentially produce more beneficial effects on student outcomes than interventions that focus exclusively on the student or individual (McNeely et al., 2002).

\section{Supporting information}

Additional supporting information may be found online in the Supporting Information section at the end of the article:

Table S1. Descriptive statistics by full sample and by externalizing trajectories.

Table S2. Bivariate correlations.

Table s3. Fit statistics for the latent class growth (LCG) analysis.

Table S4. Average latent class probabilities for the most likely latent class membership (row) by latent class (column).

Table S5. Multinomial logistic regressions predicting adult externalizing behavior trajectories (with Normal trajectory as the reference group).

Table S6. Sensitivity analysis of the indirect effects of phenotypic and polygenic models of ADHD through the simultaneous effects of adolescent sensation seeking and psychosocial mediators.

\section{Acknowledgements}

This research uses data from Add Health, a program project directed by Kathleen Mullan Harris and designed by J. Richard Udry, Peter S. Bearman, and Kathleen Mullan Harris at the University of North Carolina at Chapel Hill and funded by grant P01HD31921 from the Eunice Kennedy Shriver National Institute of Child Health and Human Development, with cooperative funding from 23 other federal agencies and foundations. No direct support was received from grant P01-HD31921 for this analysis. Special acknowledgment is due to Ronald R. Rindfuss and Barbara Entwisle for assistance in the original design. Information on how to obtain the Add Health data files is available on the Add Health website (http://www.cpc. unc.edu/addhealth). The author has declared that he has no competing or potential conflicts of interest.

\section{Correspondence}

James J. Li, Department of Psychology, University of Wisconsin-Madison, 1202 West Johnson Street, Brogden Hall, Madison, WI 53706, USA; Email: james.li@ wisc.edu 


\section{Key points}

- Childhood ADHD is associated with adult externalizing behaviors, including antisocial behaviors and substance use.

- Little is known about how this risk unfolds across development, including why some children with ADHD do not develop adult externalizing behaviors.

- Phenotypic and polygenic models of ADHD showed robust associations with High Decreasing and Moderate adult externalizing trajectories, but not Low Increasing trajectory.

- Adolescent school connectedness emerged as a robust psychosocial mechanism underlying the association of ADHD and High Decreasing and Moderate externalizing trajectories.

- Schools play a critical role in shaping the development of externalizing behaviors in adulthood, particularly among individuals with ADHD.

\section{Notes}

${ }^{1}$ The unidimensionalilty of these data were established via confirmatory factor models comparing unidimensional, two-factor, three-factor, and bifactor models across each wave of data consistently showed that bifactor solutions (specifying a general factor and three secondary factors) were a superior fit to the data. Results (including factor loadings and fit indices) are available upon request of the author. ${ }^{2}$ Analyses were also conducted where predictor variables were regressed on class probability vectors (separately for each class) derived from the best fitting LGC models to account for individual differences in classification error. Results from these models were entirely consistent with multinomial logistic regression models. Results available upon request.

\section{References}

Albert, D., Chein, J., \& Steinberg, L. (2013). The teenage brain: Peer influences on adolescent decision making. Current Directions in Psychological Science, 22, 114-120.

American Psychiatric Association (2013). Diagnostic and statistical manual of mental disorders (5th edn). Washington, DC: Author

Banaschewski, T., Brandeis, D., Heinrich, H., Albrecht, B., Brunner, E., \& Rothenberger, A. (2003). Association of ADHD and conduct disorder - brain electrical evidence for the existence of a distinct subtype. Journal of Child Psychology and Psychiatry, 44, 356-376.

Batanova, M., \& Loukas, A. (2014). Unique and Interactive Effects of Empathy, Family, and School Factors on Early Adolescents' Aggression. Journal of Youth and Adolescence, 43, 1890-1902.

Beauchaine, T.P., Hinshaw, S.P., \& Pang, K.L. (2010). Comorbidity of Attention-Deficit/Hyperactivity Disorder and earlyonset Conduct Disorder: Biological, environmental, and developmental mechanisms. Clinical Psychology: Science and Practice, 17, 327-336.

Bosco, G.L., Renk, K., Dinger, T.M., Epstein, M.K., \& Phares, V. (2003). The connections between adolescents' perceptions of parents, parental psychological symptoms, and adolescent functioning. Journal of Applied Developmental Psychology, 24, 179-200.

Braudt, D., \& Harris, K. (2018). Polygenic Scores (PGSs) in the National Longitudinal Study of Adolescent to Adult Health (Add Health) - Release 1. Carolina Digital Repository, https://www.cpc.unc.edu/projects/addhealth/documenta tion/guides/PGS_AH1_UserGuide.pdf

Brikell, I., Larsson, H., Lu, Y., Pettersson, E., Chen, Q., KujaHalkola, R., .. \& Martin, J. (2018). The contribution of common genetic risk variants for ADHD to a general factor of childhood psychopathology. Molecular Psychiatry, in press. https://doi.org/10.1038/s41380-018-0109-2

Burke, J.D., Pardini, D.A., \& Loeber, R. (2008). Reciprocal relationships between parenting behavior and disruptive psychopathology from childhood through adolescence. Journal of Abnormal Child Psychology, 36, 679-692.

Carbonaro, W., \& Workman, J. (2013). Dropping out of high school: Effects of close and distant friendships. Social Science Research, 42, 1254-1268.

Crosnoe, R., Erickson, K.G., \& Dornbusch, S.M. (2002). Protective functions of family relationships and school factors on the deviant behavior of adolescent boys and girls: Reducing the impact of risky friendships. Youth and Society, 33, 515-544.

Demontis, D., Walters, R.K., Martin, J., Mattheisen, M., Als, T.D., Agerbo, E., ... \& Neale, B.M. (2019). Discovery of the first genome-wide significant risk loci for attention deficit/ hyperactivity disorder. Nature Genetics, 51, 63.

Glass, K., Flory, K., \& Hankin, B.L. (2012). Symptoms of ADHD and close friendships in adolescence. Journal of Attention Disorders, 16, 406-417.

Harold, G.T., Leve, L.D., Barrett, D., Elam, K., Neiderhiser, J.M., Natsuaki, M.N., . . \& Thapar, A. (2013). Biological and rearing mother influences on child ADHD symptoms: Revisiting the developmental interface between nature and nurture. Journal of Child Psychology and Psychiatry, 54, 1038-1046.

Hayes, A.F. (2013). Introduction to mediation, moderation, and conditional process analysis: A regression-based approach. Guilford Publications. New York, NY: Guilford Press.

Heiman, T. (2005). An examination of peer relationships of children with and without Attention Deficit Hyperactivity Disorder. School Psychology International, 26, 330-339.

Jacobson, K.C., \& Rowe, D.C. (1999). Genetic and environmental influences on the relationships between family connectedness, school connectedness, and adolescent depressed mood: Sex differences. Developmental Psychology, 35, 926-939.

Jager, J., Yuen, C.X., Putnick, D.L., Hendricks, C., \& Bornstein, M.H. (2015). Adolescent-peer relationships, separation and detachment from parents, and internalizing and externalizing behaviors: Linkages and interactions. The Journal of Early Adolescence, 35, 511-537.

Knafo, A., \& Jaffee, S.R. (2013). Gene-environment correlation in developmental psychopathology. Development and Psychopathology, 25, 1-6.

Lahey, B.B., Zald, D.H., Hakes, J.K., Krueger, R.F., \& Rathouz, P.J. (2014). Patterns of heterotypic continuity associated 
with the cross-sectional correlational structure of prevalent mental disorders in adults. JAMA Psychiatry, 71, 989-996.

$\mathrm{Li}$, J.J. (2017). Assessing the interplay between multigenic and environmental influences on adolescent to adult pathways of antisocial behaviors. Development and Psychopathology, 29, 1947-1967.

Li, J.J., Berk, M.S., \& Lee, S.S. (2013). Differential susceptibility in longitudinal models of gene-environment interaction for adolescent depression. Development and Psychopathology, 25(4pt1), 991-1003. https://doi.org/10. $1017 /$ S0954579413000321.

Li, J.J., Savage, J.E., Kendler, K.S., Hickman, M., Mahedy, L., Macleod, J., . . \& Dick, D.M. (2017). Polygenic risk, personality dimensions, and adolescent alcohol use problems: A longitudinal study. Journal of Studies on Alcohol and Drugs, 78, 442-451.

Lindell, M.K., \& Whitney, D.J. (2001). Accounting for common method variance in cross-sectional research designs. Journal of Applied Psychology, 86, 114-121.

McNeely, C.A., Nonnemaker, J.M., \& Blum, R.W. (2002). Promoting school connectedness: Evidence from the National Longitudinal Study of Adolescent Health. Journal of School Health, 72, 138-146.

Moffitt, T.E., Caspi, A., Harrington, H., \& Milne, B.J. (2002). Males on the life-course-persistent and adolescence-limited antisocial pathways: Follow-up at age 26 years. Development and Psychopathology, 14, 179-207.

Mordre, M., Groholt, B., Kjelsberg, E., Sandstad, B., \& Myhre, A.M. (2011). The impact of ADHD and conduct disorder in childhood on adult delinquency: A 30 years follow-up study using official crime records. BMC Psychiatry, 11, 57.

Mrug, S., \& Windle, M. (2009). Bidirectional influences of violence exposure and adjustment in early adolescence: Externalizing behaviors and school connectedness. Journal of Abnormal Child Psychology, 37, 611-623.

Muthén, L.K., \& Muthén, B.O. (2015). Mplus User's Guide (7th edn). Los Angeles, CA: Muthén \& Muthén.

Nagin, D.S., \& Tremblay, R.E. (2005). Developmental trajectory groups: Fact or a useful statistical fiction?*. Criminology, 43, 873-904.
Neumann, A., Pappa, I., Lahey, B.B., Verhulst, F.C., MedinaGomez, C., Jaddoe, V.W., ... \& Tiemeier, H. (2016). Single nucleotide polymorphism heritability of a general psychopathology factor in children. Journal of the American Academy of Child and Adolescent Psychiatry, 55, 10381045. e4.

Price, A.L., Patterson, N.J., Plenge, R.M., Weinblatt, M.E., Shadick, N.A., \& Reich, D. (2006). Principal components analysis corrects for stratification in genome-wide association studies. Nature Genetics, 38, 904-909.

Rhee, S.H., Lahey, B.B., \& Waldman, I.D. (2015). Comorbidity among dimensions of childhood psychopathology: Converging evidence from behavior genetics. Child Development Perspectives, 9, 26-31.

Riglin, L., Collishaw, S., Thapar, A.K., Dalsgaard, S., Langley, K., Smith, G.D., ... \& Thapar, A. (2016). Association of genetic risk variants with attention-deficit/hyperactivity disorder trajectories in the general population. JAMA Psychiatry, 73, 1285-1292.

Sampson, R.J., \& Laub, J.H. (2003). Life-Course Desisters? Trajectories of crime among delinquent boys followed to age 70*. Criminology, 41, 555-592.

Shochet, I.M., Dadds, M.R., Ham, D., \& Montague, R. (2006). School connectedness is an underemphasized parameter in adolescent mental health: Results of a community prediction study. Journal of Clinical Child and Adolescent Psychology, 35, 170-179.

Storebø, O.J., \& Simonsen, E. (2016). The association between ADHD and antisocial personality disorder (ASPD): A review. Journal of Attention Disorders, 20, 815-824.

Tuvblad, C., Zheng, M., Raine, A., \& Baker, L.A. (2009). A common genetic factor explains the covariation among ADHD ODD and CD symptoms in 9-10 year old boys and girls. Journal of Abnormal Child Psychology, 37, 153-167.

Accepted for publication: 3 April 2019 